\title{
Design of an efficient terahertz wave source from a GaP waveguide embedded in a silicon slot waveguide
}

\author{
K. Saito \\ k-saito@material.tohoku.ac.jp
}

\section{T. Tanabe}

Y. Oyama

\author{
Department of Materials Science, Graduate School of Engineering, Tohoku University, Aoba-yama \\ 6-6-11-1021, Sendai 980-8579 Japan \\ Institute of Multidisciplinary Research for Advanced Materials, Tohoku University 2-1-1, Katahira, \\ Aoba-ku, Sendai, 980-8577, Japan \\ Department of Materials Science, Graduate School of Engineering, Tohoku University, Aoba-yama \\ 6-6-11-1021, Sendai 980-8579 Japan
}

Here, we propose an efficient scheme for terahertz ( $\mathrm{THz}$ ) wave generation on the basis of difference frequency mixing (DFM) using a CaP ridge waveguide embedded in a silicon slot waveguide. Phase matching in the DFM process, between the nonlinear polarisation wave induced by two near-infrared pumps and the generated THz wave in the low-refractive-index slot waveguide, was achieved by utilising the modal birefringence of the fundamental transverse electric- and transverse magnetic-like modes at telecom wavelengths in the CaP ridge waveguide. The effective cross-sectional area of the THz wave in the waveguide was small, $220 \mu \mathrm{m}^{2}$ at $2.26 \mathrm{THz}$, resulting in a photon conversion efficiency of $5.7 \times 10^{-2} \%$. The THz output power approached the multi- $\mu \mathrm{W}$ level using the proposed waveguide structure. [DOI: http://dx.doi.org/10.2971/jeos.2015.15024]

Keywords: Nonlinear optical effect, frequency mixing, semiconductor waveguide, terahertz wave

\section{INTRODUCTION}

Terahertz $(\mathrm{THz})$ sources, which operate in the wavelength range of $0.1-10 \mathrm{THz}$, have attracted a great deal of attention with regard to material science, biochemistry, security, and non-destructive testing applications; the availability of $\mathrm{THz}$ sources has advanced development in numerous areas by tuning to the specific eigenmodes of materials derived from optical phonons, inter- or intra-molecular vibration, and hydrogen bonding [1]-[6].

Nonlinear optics (NLO) facilitate efficient generation of $\mathrm{THz}$ waves, by converting near-infrared frequencies to the $\mathrm{THz}$ regime. Difference frequency mixing (DFM), in particular, has demonstrated high-power, frequency-tuneable, $\mathrm{THz}$ wave generation at room temperature [7]-[11]. Effective DFM requires strong nonlinear interaction among the pump, signal, and $\mathrm{THz}$ waves; for this to occur, the nonlinear interaction cross-section must be sufficiently small to satisfy the quantum conversion efficiency limit specified by the Manley-Rowe relation. Especially, a semi-insulating GaP single crystal is one of the promising NLO materials compared with other ones such as GaAs, GaSe, and $\mathrm{LiNbO}_{3}$ due to the following features: relatively high nonlinear optical susceptibility $(50 \mathrm{pm} / \mathrm{V}$ at $1.55 \mu \mathrm{m}$ [12]) wide transparency ranges for both infrared and $\mathrm{THz}$ regions, and low multiphoton absorption coefficient at telecom wavelength. In addition, semiconductor fabrication processes have been established, and is advantageous as $\mathrm{THz}$ optical devices.

Compared with bulk NLO crystals, THz waveguides made from NLO crystals, such as GaAs, GaP, and $\mathrm{LiNbO}_{3}$, with a cross-sectional size on the order of the $\mathrm{THz}$ wavelength considered, effectively confine the generated $\mathrm{THz}$ waves [13]-[21]. However, despite strong confinement of the generated $\mathrm{THz}$ wave through DFM processes, $\mathrm{THz}$ wave absorption is relatively large compared with that of optical waves, due to optical phonon and free-carrier absorption. High-resistivity silicon crystal-based $\mathrm{THz}$ waveguides exhibit a smaller absorption coefficient than that of NLO crystals. The cross-sectional dimensions of $\mathrm{THz}$ waveguides, however, are limited by the nearly THz-wavelength-scale, in the order of a few hundred microns.

Slot waveguides use a low-refractive index region to strongly confine optical waves, sub-wavelength in scale, via total internal reflection [22]-[25]. The high electric field amplitude in the low-index material, unattainable with conventional dielectric waveguides, enhances nonlinear interactions between the optical waves and NLOs. Slot waveguides and their operating principles have been applied to optical switching, optical amplifiers, optical detectors, and biosensing devices with integrated silicon photonic circuits [26]-[30]. Slot waveguides are expected to facilitate efficient $\mathrm{THz}$ wave generation, due to their light-confinement capabilities and low propagation-loss properties.

In this paper, we describe a $\mathrm{GaP}$ ridge waveguide-based $\mathrm{THz}$ wave generator embedded in a silicon-slot THz-waveguide structure. Phase-matching analysis of the DFM process and estimation of the resulting $\mathrm{THz}$ conversion efficiency were conducted for the proposed waveguide structure. Utilising 
slot waveguide

(high R silicon)

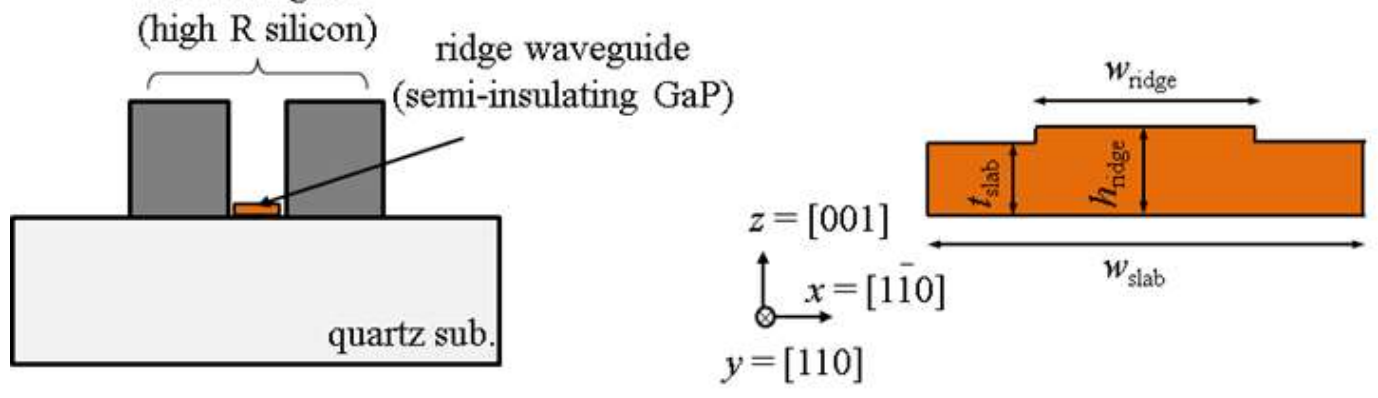

(a)

(b)

FIG. 1 (a) Cross-sectional view of a GaP-based nonlinear optic (NLO) waveguide embedded in a Si slot waveguide for terahertz (THz) wave generation via difference frequency mixing (DFM). (b) Detailed illustration of the GaP ridge waveguide structure.

the birefringence of the GaP ridge waveguide, phase matching was achieved between the nonlinear polarisation wave and resulting $\mathrm{THz}$ wave by satisfying the low group index. Strong confinement of the $\mathrm{THz}$ wave in the air-gap region of slot waveguide resulted in a high conversion efficiency, compared with that obtained using conventional dielectric waveguides.

\section{GaP-BASED NLO WAVEGUIDE EMBBED IN Si SLOT WAVEGUIDE FOR THE DFM PROCESS}

Figure 1(a) shows a schematic drawing of a Si slot waveguide, including the $\mathrm{GaP}$ ridge waveguide for $\mathrm{THz}$-wave generation. The Si slot waveguide, composed of high-resistivity silicon (cross-sectional height: $15 \mu \mathrm{m}$; cross-sectional width: $18 \mu \mathrm{m}$ ), sandwiched the GaP ridge waveguide, with a $6-\mu \mathrm{m}$ spacing. The waveguide assembly was installed on a quartz substrate. The special modal properties of the incident pump waves are defined by the ridge waveguide with cross-sectional dimensions, $h_{\text {ridge }}, w_{\text {ridge }}, t_{\text {slab }}$, and $w_{\text {slab }}$ in Figure $1(\mathrm{~b})$. In order to realize the single mode propagation of the pump waves in the ridge waveguide, these dimensions should be satisfied the following condition [31]:

$$
\frac{w_{\text {ridge }}}{h_{\text {ridge }}}<0.3+\frac{r}{\sqrt{1-r^{2}}}, \quad r=\frac{t_{\text {slab }}}{h_{\text {ridge }}}, \quad r>0.5
$$

According to Eq. (1), we set the ridge waveguid dimensions, $h_{\text {ridge, }}, w_{\text {ridge }}, t_{\text {slab }}$ and $w_{\text {slab }}$ to be $1.0 \mu \mathrm{m}, 1.2 \mu \mathrm{m}, 0.8 \mu \mathrm{m}$ and $3.0 \mu \mathrm{m}$, respectively.

The fabrication process for the proposed waveguide is as follows. A GaP wafer was optically bonded to a single-crystal quartz substrate using direct wafer bonding. The thickness of the GaP layer was reduced by chemical mechanical polishing (CMP). Photolithography and reactive ion etching (RIE) were used to construct the ridge waveguide structure in GaP. The Si slot waveguide, fabricated using RIE, was bonded to the quartz substrate.

Figure 2 shows the effective refractive index $n_{\text {eff }}$, and respective mode attenuation coefficient $n_{\text {eff }}$, of the slot waveguide as a function of $\mathrm{THz}$ frequency. Calculations were conducted using a finite-difference mode solver [32]. In the calculations, we took into account the refractive index and material attenuation at $\mathrm{THz}$ frequencies for $\mathrm{GaP}$, silicon, and quartz. The

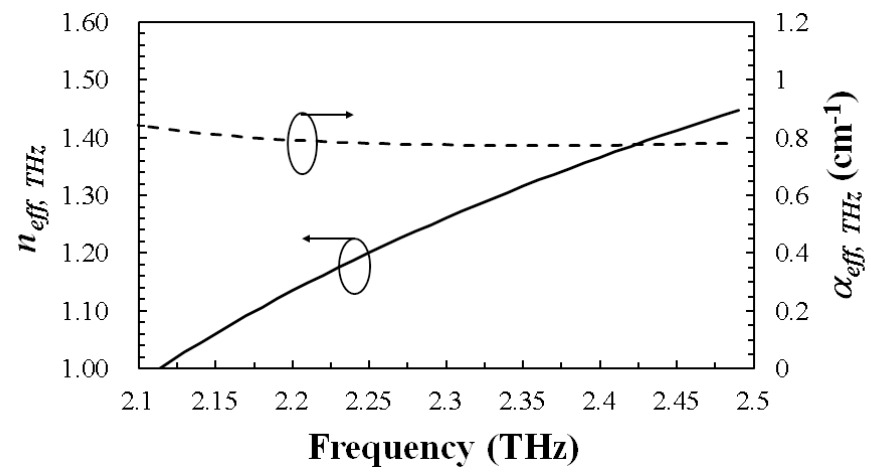

FIG. 2 Modal effective index and effective attenuation coefficient $n_{\text {eff, }}$ THz and $\alpha_{\text {eff, }}$, THz for fundamental transverse electric (TE)-like THz-waves in the slot waveguide as a function of THz frequency.

complex refractive indices of GaP, silicon, and quartz, $n+i \kappa$, at $2.26 \mathrm{THz}$ are $3.3615+0.002144 i, 3.4180+0.0004974 i$, and $2.1406+0.00093 i$, respectively $[33,34]$.

The numerically estimated effective index $n_{\text {eff }}$ and modal attenuation coefficient $n_{\text {eff }}$ are shown in Figure 2 . The small refractive indices values, characteristic of the slot waveguide, were confirmed. The calculated refractive index of the slot waveguide was 1.366 at $2.4 \mathrm{THz}$, significantly smaller than that of the $\mathrm{GaP}$ core region (3.3 at $2 \mathrm{THz}$ [35]); thus, the $\mathrm{THz}$ wave should be confined to the gap region of the slot waveguide, with a low modal attenuation coefficient of $0.77 \mathrm{~cm}^{-1}$ at $2.4 \mathrm{THz}$.

To satisfy phase-matching conditions among the optical pump, signal wave, and generated $\mathrm{THz}$ wave, we utilised modal birefringence of the $\mathrm{GaP}$ ridge waveguide. Two incident optical pump and signal waves, having frequencies of $\omega_{p}$ and $\omega_{s}$, respectively, propagated collinearly along the [110] crystalline direction of the GaP ridge waveguide shown in Figure 1. When the polarisation directions of the pump and signal waves were set parallel with respect to the [001] (transverse magnetic (TM) mode) and [110] (transverse electric (TE) mode) directions, the group index of the induced nonlinear polarisation wave was reduced, compared with the refractive index of $\mathrm{GaP}$, resulting in the generation of TE-like $\mathrm{THz}$ wave confined in the slot waveguide.

Figure 3(a) shows the calculated modal indices of the TE and $\mathrm{TM}$ modes in a $\mathrm{GaP}$ ridge waveguide. We confirmed a large modal birefringence of $\Delta n \approx 0.022$ between the TE and TM modes in the $\mathrm{GaP}$ ridge waveguide. From these modal disper- 


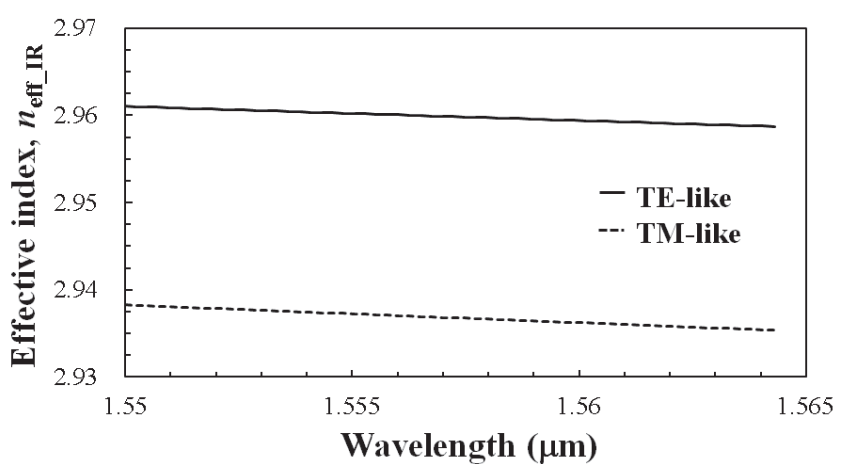

(a)

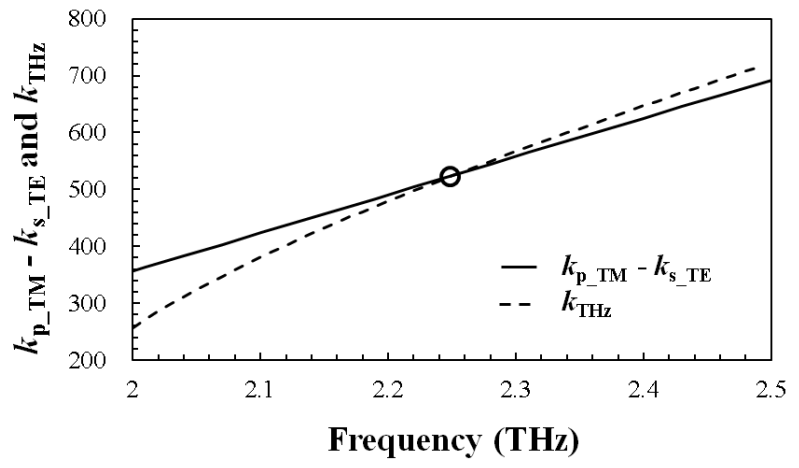

(b)

FIG. 3 (a) Modal effective indices for fundamental TE-like and transverse magnetic (TM)-like modes in the GaP ridge waveguide in the telecom wavelength region. (b) Propagation constant for the nonlinear polarisation wave generated by the optical waves $\beta_{\mathrm{IR}}$, and guided THz wave in the slot waveguide $\beta_{T H z}$, as a function of the difference frequency between the pump and signal waves. The open circle highlighted in Figure 3 (b) indicates the phase-matching frequency position.

sions, the group index of the nonlinear polarisation wave, $n_{g}$, was estimated in Figure 3(b) for a fixed pump wavelength of $1.55 \mu \mathrm{m}$. The large modal index difference between the fundamental TM-like and TE-like modes produced a nonlinear polarisation wave with a low group index. The propagation constant of the nonlinear polarisation wave, generated by the pump and signal waves, is given by:

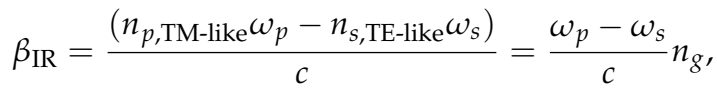

where $\omega_{i}(i=p$ and $s)$ are the angular frequencies of the pump and signal waves. The modal indices for the fundamental TM-like pump and TE-like signal waves, $n_{p, \text { TM-like }}$ and $n_{s, \mathrm{TE}-\mathrm{like}}$, provide a group index for the nonlinear polarisation wave $n_{g}$. The propagation constant of the nonlinear polarisation wave $\beta_{\mathrm{IR}}$ is shown in Figure 3(b), as well as that for the guided $\mathrm{THz}$ wave in the slot waveguide obtained from the effective index $n_{\text {eff,THz }}$. The frequency position, highlighted by an open circle, indicates that the phase-matching condition is satisfied at $2.26 \mathrm{THz}$ for the designed waveguide structure.

Here, we consider the propagation losses in the waveguide associated with the phase-matching conditions for the DFM process. Figure 4(a) illustrates the normalized optical intensity distribution of the TE-like pump wave at $1.5686 \mu \mathrm{m}$ in the GaP ridge waveguide. The optical wave is confined into the ridge waveguide. The propagation losses for the optical wave were estimated by beam propagation method. Figure 4(a) shows the optical intensity change as a function of the ridge waveguide length. From this figure, the propagation loss in the ridge waveguide $\beta_{\mathrm{IR}}$ was estimated to be $1.05 \mathrm{~cm}^{-1}$.

We introduced a complex phase-mismatch factor associated with DFM, as follows:

$$
\Delta \beta=\Delta \beta_{R}+i \Delta \beta_{I}=\left(\beta_{\mathrm{IR}}-\beta_{\mathrm{THz}}\right)+i\left(\alpha_{\mathrm{IR}}-\frac{\alpha_{\mathrm{eff}, \mathrm{THz}}}{2}\right)
$$

where $\Delta \beta_{R}$ and $\Delta \beta_{I}$ denote the real and imaginary parts of the phase-mismatch term. The imaginary part of Eq. (3) restricts the $\mathrm{THz}$-wave power as it propagates along the waveguide. The coherence length $L_{c}$ in the DFM process satisfies the figure of merit in the designed NLO device, with the optimal length designed for maximum output power. The coherence length is calculated from the following equation [36]:

$$
\begin{aligned}
-\left(\alpha_{\mathrm{eff}, \mathrm{THz}}+\Delta \beta_{I}\right)\left[\cosh \left(\Delta \beta_{I} L_{c}\right)-\cos \left(\Delta \beta_{R} L_{c}\right)\right] \\
+\Delta \beta_{I} \sinh \left(\Delta \beta_{I} L_{c}\right)+\Delta \beta_{R} \sin \left(\Delta \beta_{R} L_{c}\right)=0
\end{aligned}
$$

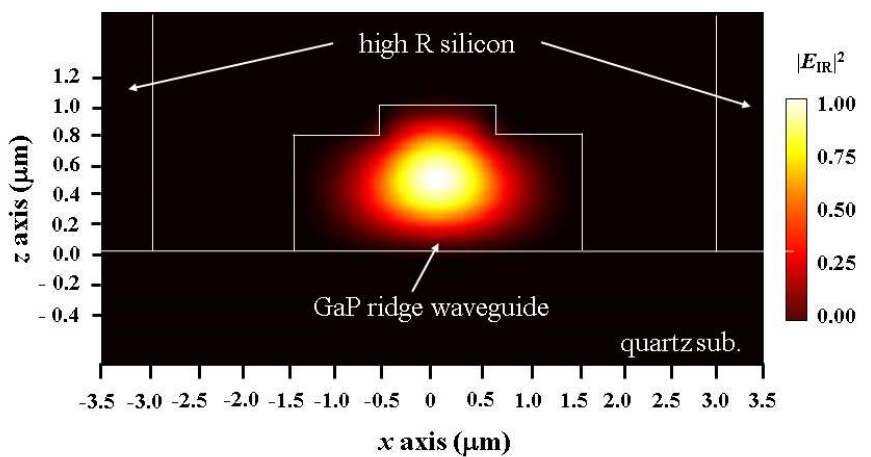

(a)

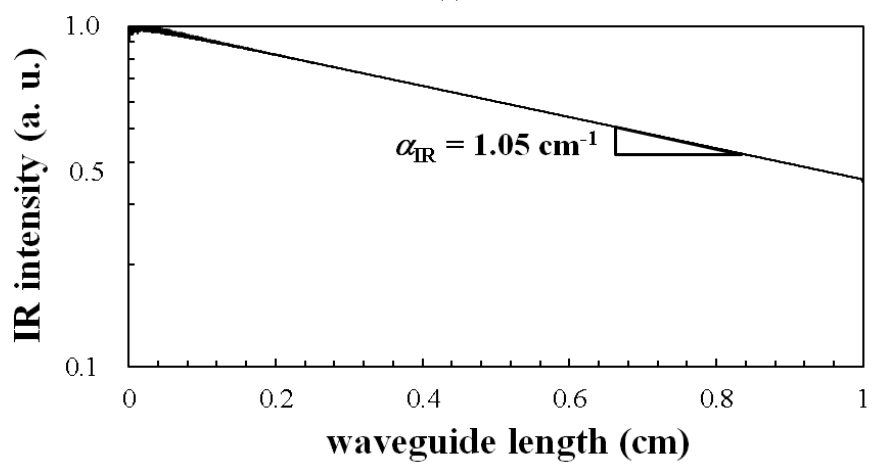

(b)

FIG. 4 (a) Normalised optical intensity distribution of the TE-like pump wave at $1.5686 \mu \mathrm{m}$ in the GaP ridge waveguide. (b) The optical power change as a function of the waveguide length. The propagation loss was estimated to be $1.05 \mathrm{~cm}^{-1}$.

Numerical estimation of the coherence length for $2.26-\mathrm{THz}$ generation was $1.4 \mathrm{~cm}$, where the phase-mismatch terms $\Delta \beta_{R}$ and $\Delta \beta_{I}$, were $0 \mathrm{~cm}^{-1}$ and $0.67 \mathrm{~cm}^{-1}$, respectively.

\section{THZ OUTPUT CHARACTERISTICS IN THE DFM PROCESS}

The conversion efficiency in the DFM process for the GaPbased slot waveguide was estimated using the following [37]:

$$
\begin{aligned}
& \eta_{\mathrm{THz}}\left[W^{-}\right]=\frac{P_{\mathrm{THz}}}{P_{p} P_{S}}=\omega_{\mathrm{THz}}^{2} \Gamma^{2} L^{2} \\
& \cdot \exp \left[-\left(\alpha_{\mathrm{eff}, \mathrm{THz}}+\Delta \beta_{I}\right) L\right] \frac{\cosh ^{2}\left(\Delta \beta_{I} L / 2\right)-\cos \left(\Delta \beta_{R} L\right)}{\left(\Delta \beta_{R} L / 2\right)^{2}+\left(\Delta \beta_{I} L / 2\right)^{2}}
\end{aligned}
$$

where $P_{i}$ and $\alpha_{i}(i=p, s$, and $\mathrm{THz})$ are the optical power and absorption coefficients for the pump, signal, and THz waves, 
respectively, and $L$ is the waveguide length. $\Gamma$ is the nonlinear coupling coefficient, given by:

$$
\Gamma^{2}=d_{q}^{2}\left(\frac{2 \mu_{0}}{c n_{p} n_{s} n_{\mathrm{THz}} A_{\mathrm{eff}}}\right)
$$

where $d_{q}$ is the second-order nonlinear coefficient of GaP, $n_{i}$ ( $i=p, s$, and $\mathrm{THz}$ ) are the refractive indices of the pump, signal, and $\mathrm{THz}$ waves, respectively, $c$ is the speed of light, and $\mu_{0}$ is the permeability in vacuum.

The second-order nonlinear coefficient of $\mathrm{GaP}, d_{q}$, is expressed by [38]:

$$
d_{q}=d_{e}\left(1-0.53 \frac{v_{\mathrm{TO}}^{2}}{v_{\mathrm{TO}}^{2}-v_{\mathrm{THz}}^{2}-i \gamma \nu_{\mathrm{THz}}}\right),
$$

where $d_{e}$ is the electronic second-order nonlinear coefficient $\left(d_{e}=50 \mathrm{pm} \mathrm{V}^{-1}\right.$ [12]), and $v_{\mathrm{TO}}$ and $\gamma$ correspond to the transverse optical $(\mathrm{TO})$ phonon frequency $\left(v_{\mathrm{TO}}=11.01 \mathrm{THz}\right)$ and its damping constant $(\gamma=0.03 \mathrm{THz})$. The effective nonlinear interaction area $A_{\text {eff }}$ is given by:

$$
A_{\mathrm{eff}}=\frac{\int\left|E_{p}\right|^{2} d x d z \int\left|E_{s}\right|^{2} d x d z \int\left|E_{\mathrm{THz}}\right|^{2} d x d z}{d_{q}^{-2}\left|\int d_{q}(x, z) E_{p}^{*} E_{s} E_{\mathrm{THz}} d x d z\right|^{2}},
$$

where $E_{i}(i=p, s$, and $T H z)$ are the normalised complex amplitudes of the electric field of the pump, signal, and $\mathrm{THz}$ waves, respectively. Figure 5 shows the normalised electric field distribution of the $\mathrm{THz}$ wave in the slot waveguide at $2.26 \mathrm{THz}$ (Figure 5(a)) and effective nonlinear interaction area $A_{\text {eff }}$ expressed by Eq. (8) (Figure 5(b)). The effective area $A_{\text {eff }}$ was estimated to be $\sim 220 \mu \mathrm{m}^{2}$, resulting in a coupling coefficient $\Gamma$ of $3.4 \times 10^{-14}\left(\mathrm{~s} / \mathrm{m} \mathrm{W}^{-1 / 2}\right)$. The estimated value was twice lager than the area of slot region $\left(90 \mu \mathrm{m}^{2}\right)$ because the THz electric field overlaps the GaP ridge waveguide partially. The propagation loss of the slot waveguide for the $\mathrm{THz}$ wave $\alpha_{\text {eff,THz }}$ is given by:

$$
A_{\mathrm{eff}, \mathrm{THz}}=\frac{\iint E_{\mathrm{THz}}^{2}(x, y) \alpha_{\mathrm{THz}}(x, y) d x d y}{\iint E_{\mathrm{THz}}^{2}(x, y) d x d y},
$$

where $E(x, y)$ is the electric field profile of the waveguide, and $\alpha(x, y)$ is the absorption loss distribution for the entire waveguide. $\alpha_{\text {eff,THz }}$ was estimated to be $0.78 \mathrm{~cm}^{-1}$ using the absorption coefficients of $\mathrm{GaP}, \mathrm{Si}$, and quartz at $2.26 \mathrm{THz}$ : $0.5,4$, and $0.3 \mathrm{~cm}^{-1}$, respectively. From this, the $\mathrm{THz}$ output power through the DFM process as a function of the waveguide length $L$ was calculated, as shown in Figure 6. In this calculation, the power of the continuous wave $(\mathrm{CW})$ pump and signal was assumed to be $1 \mathrm{~W}$. The $\mathrm{THz}$ power increased monotonously with the waveguide length, reaching a maximum CW-THz wave power of $6.6 \mu \mathrm{W}$ when the waveguide length was $1.5 \mathrm{~cm}$, in agreement with the coherence length.

The power conversion efficiency was estimated at $6.5 \times 10^{-4} \%$, corresponding to a photon conversion efficiency of $5.7 \times 10^{-2} \%$. This value was much higher than that of the experimental results reported previously $\left(\eta=1.3 \times 10^{-5} \%\right)$ [17]. The $\mathrm{THz}$ output power was comparable to that produced by a quantum cascade-based DFM source [39]. THz power exceeding the multi- $\mu \mathrm{W}$ level can be detected using a $\mathrm{THz}$

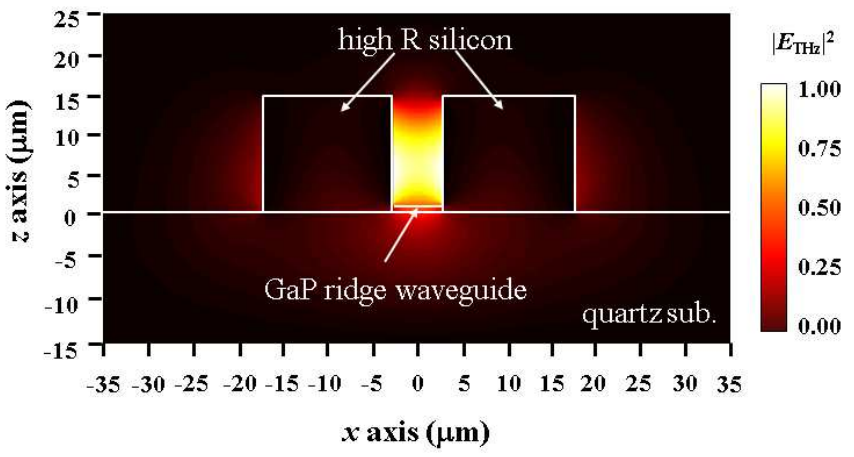

(a)

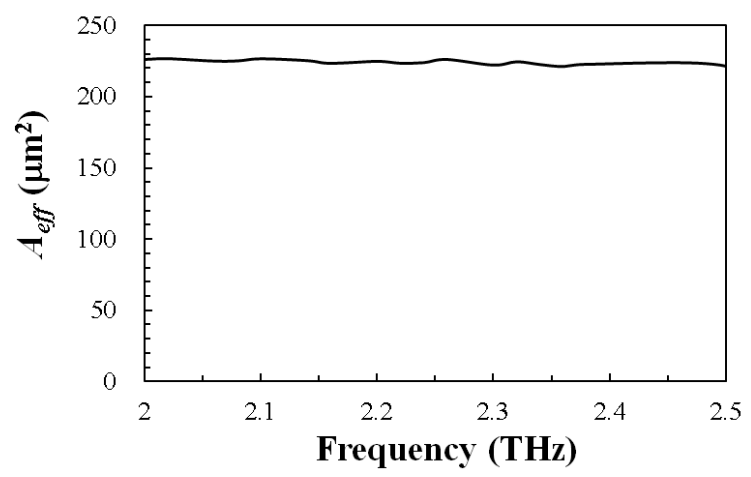

(b)

FIG. 5 (a) Normalised optical intensity distribution of the TE-like THz wave at $2.26 \mathrm{THz}$ in the slot waveguide. (b) The nonlinear interaction area, $A_{\text {eff }}$ obtained from Eq. (8). The estimated effective area $A_{\text {eff }}$ was $220 \mu \mathrm{m}^{2}$.

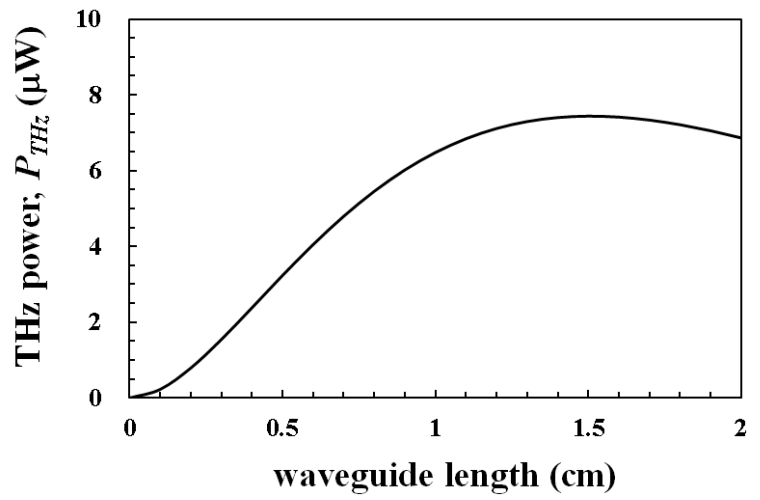

FIG. $6 \mathrm{THz}$ output power as a function of the waveguide length $L$. The input power of the pump and signal waves was fixed at $1 \mathrm{~W}$, respectively. The maximum output power was obtained to be $6.6 \mu \mathrm{W}$, which corresponds to a photon-conversion efficiency of $5.7 \times 10^{-2} \%$

detector operating at room temperature, such as a pyroelectric detector or Golay cell. Thus, the proposed waveguide structure described can be used as a compact, high-power $\mathrm{THz}$ source.

\section{CONCLUSION}

We demonstrated efficient $\mathrm{THz}$ wave generation from a GaPbased ridge waveguide embedded in a Si slot waveguide. Strong confinement and low propagation loss for the generated $\mathrm{THz}$ waves resulted in a $\mathrm{THz}$ output power of $6.6 \mu \mathrm{W}$ at $2.26 \mathrm{THz}$, corresponding to a photon conversion efficiency of $5.7 \times 10^{-2} \%$. The proposed waveguide structure is an effective way to produce high-power $\mathrm{CW}-\mathrm{THz}$ waves above the multi$\mu \mathrm{W}$ level that can be detected by $\mathrm{THz}$ detectors operating at 
room temperature, e.g., Golay cells and pyroelectric detectors. The high-power $\mathrm{THz}$ source contributes to the realisation of spectroscopic applications with high spectral resolution and wide dynamic range. Furthermore, our proposed THz device will advance the development of $\mathrm{THz}$ sources that bridge the telecom- and $\mathrm{THz}$-frequency regions.

\section{References}

[1] M. Tonouchi, "Cutting-edge terahertz technology," Nat. Photon. 1, 97-105 (2007).

[2] B. Ferguson, and X. C. Zhang, "Materials for terahertz science and technology," Nat. Mater. 1, 26-33 (2002).

[3] A. G. Markelz, A. Roitberg, and E. J. Heilweil, "Pulsed terahertz spectroscopy of DNA, bovine serum albumin (BSA) and collagen between 0.1 and 2.0 THz," Chem. Phys. Lett. 320, 42-48 (2000).

[4] S. Ohno, A. Hamano, K. Miyamoto, C. Suzuki, and H. Ito, "Surface mapping of carrier density in a GaN wafer using a frequency-agile THz source," J. Eur. Opt. Soc.-Rapid 4, 09012 (2009).

[5] K. Kawase, Y. Ogawa, Y. Watanabe, and H. Inoue, "Nondestructive terahertz imaging of illicit drugs using spectral fingerprints," Opt. Express 11, 2549-2554 (2003).

[6] P. U. Jepsen, D. G. Coole, and M. Koch, "Terahertz spectroscopy and imaging - Modern techniques and applications," Laser Photon. Rev. 5, 124-166 (2011).

[7] T. Tanabe, K. Suto, J. Nishizawa, T. Kimura, and K. Saito, "Frequency-tunable high-power terahertz wave generation from GaP," J. Appl. Phys. 93, 4610-4615 (2003).

[8] K. Kawase, M. Mizuno, S. Sohoma, H. Takahashi, T. Taniuchi, Y. Urata, S. Wada, et al., "Difference-frequency terahertzwave generation from 4-dimethylamino-N-methyl-4-stilbazoliumtosylate by use of an electronically tuned Ti:sapphire laser," Opt. Lett. 24, 1065-1067 (1999).

[9] J. Nishizawa, T. Tanabe, K. Suto, Y. Watanabe, T. Sasaki, and Y. Oyama, "Continuous-wave frequency-tunable terahertz-wave generation from GaP," IEEE Photon. Technol. Lett. 18, 2008-2010 (2006).

[10] I. Tomita, H. Suzuki, H. Ito, H. Takenouchi, K. Ajito, R. Rungsawang, and Y. Ueno, "Terahertz-wave generation from quasiphase-matched GaP for $1.55 \mu \mathrm{m}$ pumping," Appl. Phys. Lett. 88, 071118 (2006).

[11] K. L. Vodopyanov, J. E. Schaar, P. S. Kuo, M. M. Fejer, X. Yu, J. S. Harris, V. G. Kozlov, et al."Terahertz wave generation in orientation-patterned GaAs using resonantly enhanced scheme," Proc. SPIE 6455, 645509 (2007).

[12] I. Shoji, T. Kondo, A. Kitamoto, M. Shirane, and R. Ito, "Absolute scale of second-order nonlinear-optical coefficients," J. Opt. Soc. Am. B 14, 2268-2294 (1997).

[13] K. Saito, T. Tanabe, Y. Oyama, K. Suto, and J. Nishizawa, "Terahertzwave generation by $\mathrm{GaP}$ rib waveguides via collinear phasematched difference-frequency mixing of near-infrared lasers," J. Appl. Phys. 105, 063102 (2009).

[14] H. Cao, R. A. Linke, and A. Nahata, "Broadband generation of terahertz radiation in a waveguide," Opt. Lett. 29, 1751-1753 (2004).

[15] S. Coleman, and D. Grischkowsky, "Parallel plate THz transmitter," Appl. Phys. Lett. 84, 654-656 (2004).

[16] G. Chang, C. J. Divin, J. Yang, M. A. Musheinish, S. L. Williamson, A. Galvanauskas, and T. B. Norris, "GaP waveguide emitters for high power broadband THz generation pumped by $\mathrm{Yb}$-doped fiber lasers," Opt. Express 15, 16308-16315 (2007).

[17] C. Staus, T. Kuech, and L. McCaughan, "Continuously phasematched terahertz difference frequency generation in an embedded-waveguide structure supporting only fundamental modes," Opt. Express 16, 13296-13303 (2008).

[18] K. Suizu, K. Koketsu, T. Shibuya, T. Tsutsui, T. Akiba, and K. Kawase, "Extremely frequency-widened terahertz wave generation using Cherenkov-type radiation," Opt. Express 17, 6676-6681 (2009).

[19] Y. H. Avetisyan, "Terahertz-wave surface-emitted differencefrequency generation without quasi-phase-matching technique," Opt. Lett. 35, 2508-2510 (2010).

[20] K. Saito, T. Tanabe, and Y. Oyama, “Polarization selective terahertz-wave generation from GaP ridge waveguide under collinear phase-matched difference-frequency mixing," Jpn. J. Appl. Phys. 53, 102102 (2014).

[21] K. Saito, T. Tanabe, and Y. Oyama, "THz-wave generation from GaP THz photonic crystal waveguides under difference-frequency mixing," Opt. Photon. J. 2, 201-205 (2012)

[22] V. R. Almeida, Q. Xu, C. A. Barrios, and M. Lipson, "Guiding and confining light in void nanostructure," Opt. Lett. 29, 1209-1211 (2004).

[23] Q. Xu, V. R. Almeida, R. R. Panepucci, and M. Lipson, “Experimental demonstration of guiding and confining light in nanometer-size low-refractive-index material," Opt. Lett. 29, 1626-1628 (2004).

[24] R. Sun, P. Dong, N.-N. Feng, C.-Y. Hong, J. Michel, M. Lipson, and L. C. Kimerling, "Horizontal single and multiple slot waveguides: optical transmission at $\lambda=1550 \mathrm{~nm}$," Opt. Express 15, 17967 (2007).

[25] M. Nagel, A. Marchewka, and H. Kurz, "Low-index discontinuity terahertz waveguides," Opt. Express 14, 9944-9954 (2006).

[26] T. Baehr-Jones, M. Hochberg, G. Wang, R. Lawson, Y. Liao, P. A. Sullivan, L. Dalton, et al., "Optical modulation and detection in slotted silicon waveguides," Opt. Express 13, 5216-5226 (2005).

[27] C. A. Barrios, and M. Lipson, "Electrically driven silicon resonant light emitting device based on slot-waveguide," Opt. Express 13, 10092-10101 (2005).

[28] C. A. Barrios, "High-performance all-optical silicon microswitch," Electron. Lett. 40, 862-863 (2004).

[29] T. Baehr-Jones, M. Hochberg, G. Wang, R. Lawson, Y. Liao, P. A. Sullivan, L. Dalton, et al., "Optical modulation and detection in slotted silicon waveguides," Opt. Express 13, 5216-5226 (2005).

[30] C. A. Barrios, K. B. Gylfason, B. Sánchez, A. Griol, H. Sohlström, M. Holgado, and R. Casquel, "Slot-waveguide biochemical sensor," Opt. Lett. 32, 3080-3082 (2007).

[31] J. Xia, J. Yu, Y. Li, and S. Chen, "Single-mode condition for silicon rib waveguides with large cross sections," Opt. Eng. 43, 1953-1954 (2004).

[32] A. B. Fallahkhair, K. S. Li. and T. E. Murphy, "Vector finite difference modesolver for anisotropic dielectric waveguides," J. Lightwave Technol. 26, 1423-1431 (2008).

[33] E. V. Loewenstein, D. R. Smith, and R. L. Morgan, "Optical constants of far infrared materials. 2: Crystalline solids," Applied Optics 12, 398-406 (1973).

[34] K. Saito, T. Tanabe, Y. Oyama, K. Suto, T. Kimura, and J. Nishizawa, "Terahertz-wave absorption in GaP crystals with different carrier densities," J. Phys. Chem. Solids 69, 597-600 (2008). 
[35] E. D. Palik (ed.), Handbook of optical constants of solids (Academic Press, New York, 1985).

[36] V. A. Felipe, and L. M. Hayden, "Simplified model for optical rectification of broadband terahertz pulses in lossy waveguides including a new generalized expression for the coherence length," Opt. Express 21, 24398-24412 (2013).

[37] R. W. Boyd, Nonlinear optics (Third edition, Academic Press, New York, 2008).
[38] W. L. Faust, and C. H. Henry, "Mixing of visible and near-resonance infrared light in GaP," Phys Rev. Lett. 17, 1265-1268 (1966).

[39] Q. Y. Lu, N. Bandyopadhyay, S. Slivken, Y. Bai, and M. Razeghi, "Continuous operation of a monolithic semiconductor terahertz source at room temperature," Appl. Phys. Lett. 104, 221105 (2014). 